\title{
An inexpensive blackbody model
}

\author{
M. Fidali*, M. Mikulski*
}

*Department of Fundamentals of Machinery Design - Silesian University of Technology, Gliwice, Poland

\begin{abstract}
A blackbody is a fundamental concept in thermography. A perfect model of blackbody is characterized by emissivity $\varepsilon=1$ which is difficult to be obtained. There are black body simulators which are being used for calibration of infrared devices. Commercial solutions of blackbody simulators are based on mathematical models of cavity or surface blackbodies and their emissivity is closer to unity e.g. $\varepsilon=0,9 x-0,99 x[2,3,4]$ where $x$ is an integer equal to less than 9. A common feature of commercial devices is a high price. There is a concept of the blackbody based on conical geometry [1] which can be used in black body simulators. In the article practical realization of inexpensive blackbody simulator based on the radiator with conical geometry was presented. The device has been built with the use of ordinary materials and computer parts. A simple temperature controller based on microprocessor Atmega8 has been able to operate with four programmable 1-Wire Digital thermometers which are located on external surface of the cone. The controller has allowed changes of temperature in a range from room temperature to $80^{\circ} \mathrm{C}$. Results of preliminary tests of designed blackbody simulator have shown that obtained parameters have been acceptable and they correspond to computational values. Its emissivity in the whole temperature range were higher than 0,96 . Temperature distribution on bottom surface of the cone was flat. Total cost of the device was about 50 euros.
\end{abstract}

\section{Introduction}

A blackbody is an object that absorbs electromagnetic radiation totally regardless of its direction and wavelength. The blackbody has also ability to emit the energy thus it is considered as a perfect radiator. Due to its properties the blackbody has great importance in a theory of infrared measurements. There are different mathematical concepts of blackbodies which are useful in designing of its physical models. Physical models of blackbodies differ from theoretical one. Therefore a technically correct name for such device is "blackbody simulator" [4]. On the market there are commercial blackbody simulators based on principles of cavity or surface radiators. They are required in industry as reference sources for source-based calibration and calibration verification of Infrared Radiation Thermometers, Optical Pyrometers, Infrared line-measuring thermometers and Area-measuring thermometers or Quantitative Thermal Imagers. In laboratory research, they are parts of benches for characterisation of complex optronic systems $[3,4]$.

One of the key parameters of blackbody simulators is emissivity which also determines a price of such device. The emissivity of commercial blackbody simulators is measured in 9's e.g. two nines means an emissivity of $0.99 x$, three nines means emissivity $0.999 x$ etc. where $x$ is an integer less than 9 . An emissivity error can be translated readily into a radiance error during calibration. Other typical parameters of blackbody simulators are grayness, or the uniformity of its spectral emissivity with wavelength, entrance aperture diameter (usually the larger the better) and response time which is the time required to stabilize the device at a new temperature after a change in settings, prior to testing [4]. Commercial devices are expensive and some researchers which need such reference source of high emissivity can not afford to buy it.

In measurement practice there are different often sufficient methods of obtaining high emissivity sources. An example is black painting of the observed surface, drilling holes in investigated objects or using hand made simple blackbody simulators.

To design and manufacture a not expensive and accurate black body simulator with high emissivity (at least 0,95 ) in the wide range of temperatures, is not a simple task. The best emissivity value of the blackbody simulator can be obtain if the cavity radiator is used. However, in such case it is important that it provides uniform temperature on whole radiator surface, what can be a barrier in realization of such devices.

During designing a blackbody simulator spherical, conical or cylindrical geometry of the radiator can be taken into account. In [1] there was shown how to design a blackbody on the basis of conical geometry. There has been proved in analytical way that using ordinary materials having rather common emissivity $\varepsilon=0,9$ one can make a reference source with an emissivity to a value close to unity.

In the article a practical realization of a cheap blackbody simulator based on the radiator with conical geometry was presented.

\section{An idea of a blackbody with conical geometry}

An Idea of a blackbody can be based on heat transfer by radiation between grey bodies, arranged in cone geometry. An easy physical explanation for the cone is to consider radiation falling on its surface (figure 1a). Even when a material is characterized by emissivity equal to only 0.9 , or reflection equal to 0.1 , the probability than an incident light ray is reflected is much less than 0.1 [1]. It is a result of multiple reflections which take place inside the cone. In order to use a blackbody simulator with the cone radiator, an infrared device should be placed in front of the hole and focused on a virtual bottom surface of the cone. The infrared camera records a circular object characterized by temperature $T$ and emissivity $\varepsilon_{\text {app }}$ which is very close to unity. Emissivity $\varepsilon_{\text {app }}$ of the considered cone geometry shown in figure $1 \mathrm{~b}$ can be calculated with the use of equation (1) [1]: 


$$
\varepsilon_{a p p}=\frac{\varepsilon}{1-(1-\varepsilon) F_{2,2}}
$$

Where:

a)

$$
\begin{aligned}
& F_{2,2}=1-\frac{R}{\sqrt{H^{2}+R^{2}}}-\text { geometrical factor } \\
& \mathrm{R} \text { - radius of the cone bottom } \\
& \mathrm{H} \text { - height of the cone } \\
& \varepsilon \text { - emissivity of the inner surface of the cone }
\end{aligned}
$$

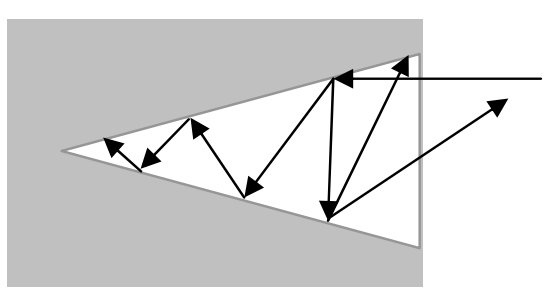

b)

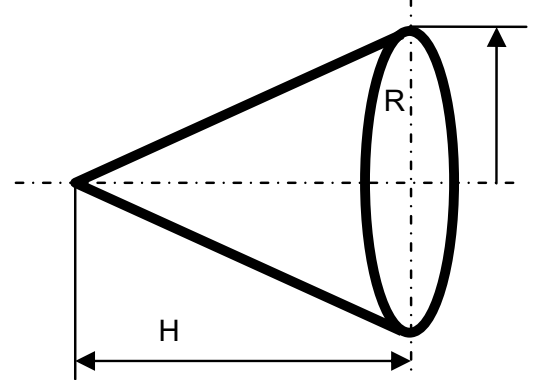

Fig. 1. An idea of a blackbody with conical geometry (a) and its geometry b)

Taking into account eqation (1) and figure $2 a$ it has been clear to observe that for assumed emissivity of the inner surface of the cone $(\varepsilon=0.9)$ a value of emissivity $\varepsilon_{\text {app }}$ tended to unity if hole diameter had tended to zero and cone height tended to infinity. From a technological point of view manufacturing a high cone with a very small aperture, which is required to obtain high emissivity, is very difficult and unjustified, because the larger aperture diameter makes such device more useful. During the designing of the cone geometry it is enough to set one parameter as constant (e.g. R) and find such value of the second parameter (e.g. $\mathrm{H}$ ), for which the value of emissivity $\varepsilon_{\text {app }}$ is sufficiently high (figure $2 \mathrm{~b}$ ). In the case of the discussed blackbody simulator one decided to manufacture the cone characterized by dimensions shown in figure $3 a$. On the one side geometrical parameters were limited by parameters of available lathe and its tools and one the other by dimensions of available enclosure of the blackbody simulator. Assumed cone dimensions allowed us to get satisfactory theoretic emissivity $\varepsilon_{\text {app }}=0,9747$.

a)

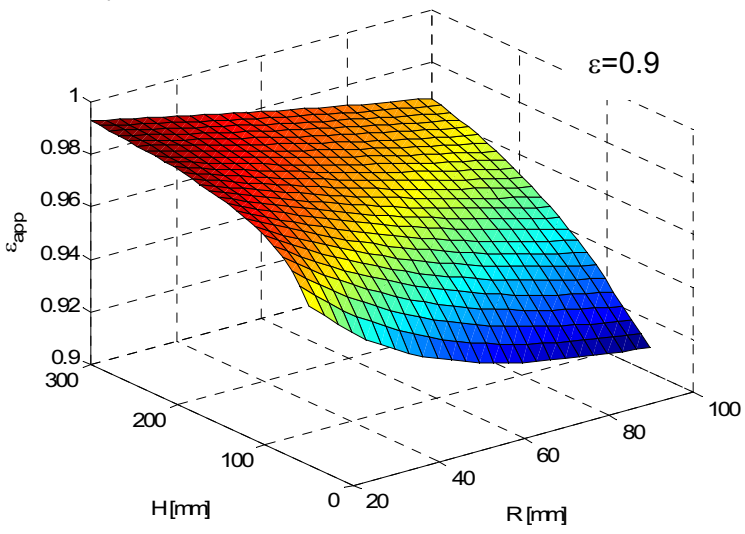

b)

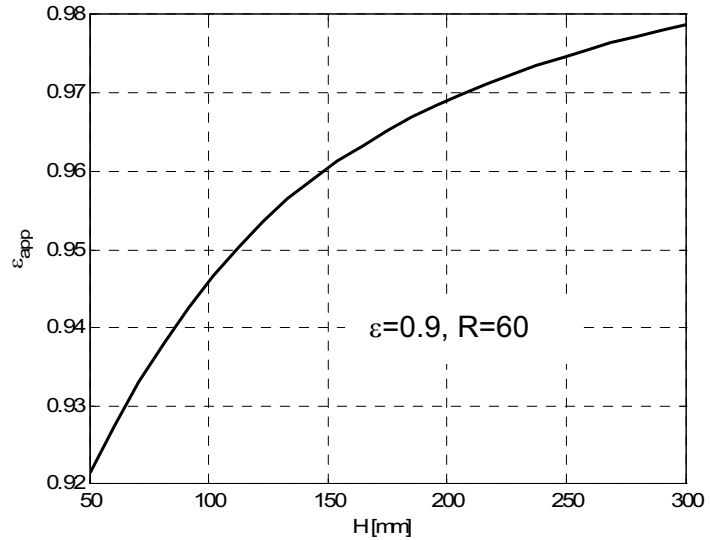

Fig. 2. Analysis of aperture emissivity changes in function of cone geometrical parameters $(R$ and $H)$ a) and in function of cone height with assumed radius $R=60 \mathrm{~mm} \mathrm{~b}$ ) for assumed emissivity of cone inner surface $\varepsilon=0.9$

a)

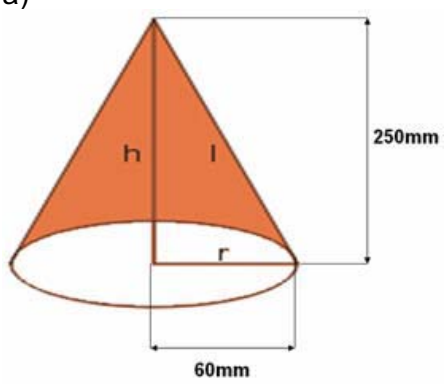

b)

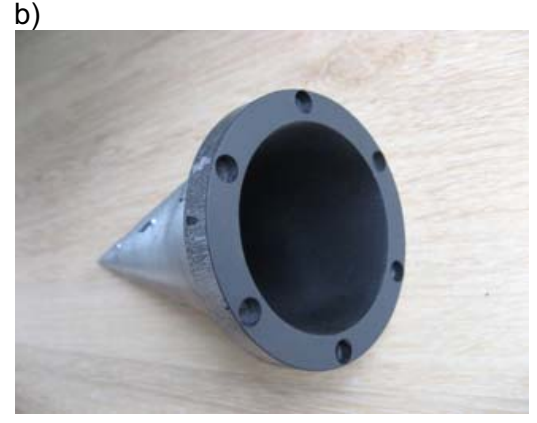

Fig. 3. Geometry of the cone radiator with its computational emissivity a) and the manufactured cone b) 


\section{Construction of the blackbody simulator}

On the basis of a concept of the radiator with conical geometry, a blackbody simulator has been designed with the use of CATIA software. During the designing it was assumed that the device is assembled from commonly accessible parts as well as parts of different second-hand devices. Thus a housing as well as some electrical parts of the simulator were taken from an old computer. In (figure 4) the assembled and ready to use blackbody simulator was presented. The device includes two fundamental parts: a heater unit and temperature controller unit. The heater unit ensures isothermal condition of the cone radiator internal surface at different temperature values which are being set on the controller. The heater unit includes a hermetic and isolated vessel, the conic radiator, an electric heater and mixer which are driven by DC motor. The vessel is filled with water which level can be controlled by means of a pipe indicator located on a rear panel of the device. The mixer has been used in order to distribute heated water into the vessel and assure uniform water temperature in all points of the vessel space. The radiator (figure $3 \mathrm{~b}$ ) has been made according to assumed geometry, and in order to get surface emissivity closer to 0,9 the internal surface of the conic radiator has been painted black.

a)

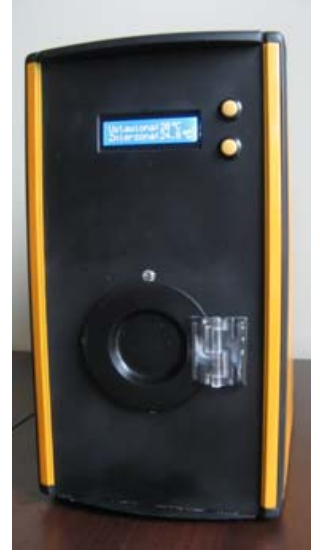

b)

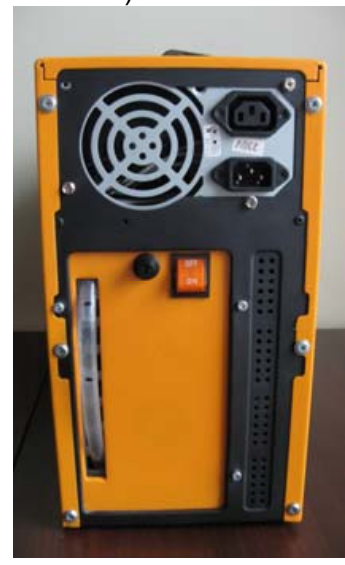

c)

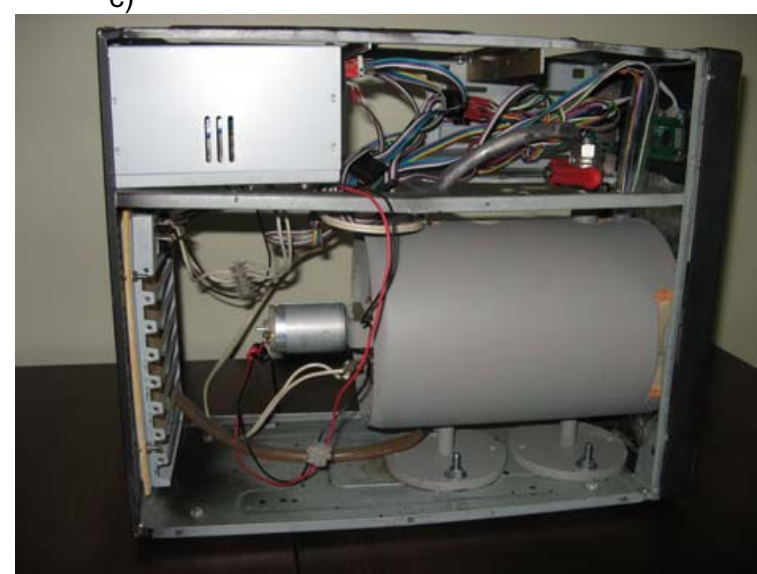

Fig. 4. View of front a) and rear b) panels of the blackbody simulator as well as its interior $\mathrm{c}$ )

To allow us precisely adjust radiator temperature in range from room temperature to $80^{\circ} \mathrm{C}$ as well as keep temperature value at the established level the heater unit has been connected to the temperature controller unit. A diagram of the temperature control system was presented in figure 5 . A fundamental part of the controller has been microprocessor Atmega8 with installed developed software. The software has included a temperature regulation heuristic algorithm which was based on data transferred from four programmable 1-Wire digital thermometers DALLAS DS18B20 located on the external surface of the conic radiator (figure 5). Parameters of the sensors have been determined parameters of the whole blackbody simulator. Additionally, the upper temperature range of the controller has been limited to $80^{\circ} \mathrm{C}$ in order to avoid water boiling in the vessel. User communication with the controller has benn performed by LCD display and two buttons which allow increasing and decreasing controlled temperature.

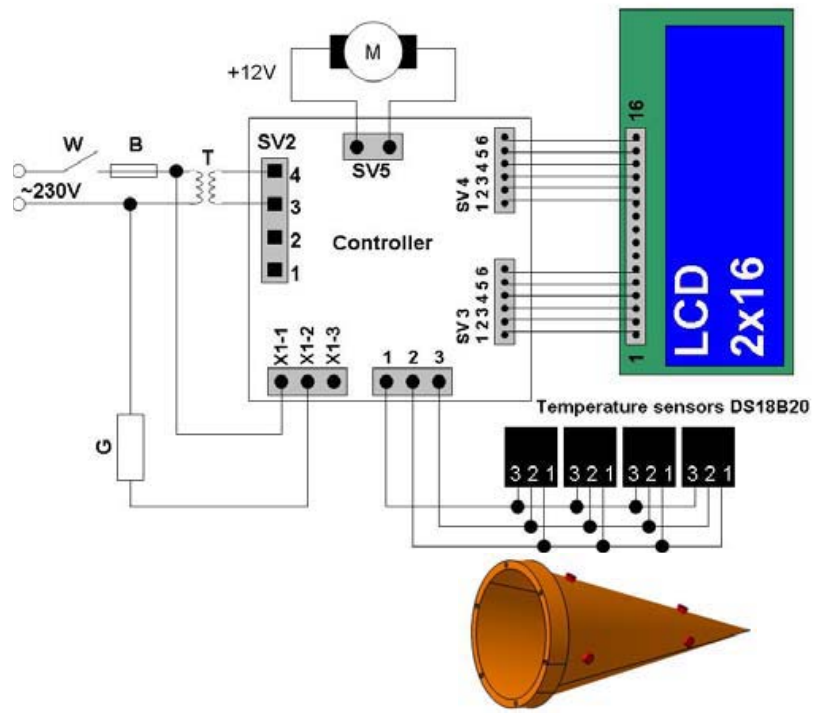

Key features of the digital thermometers applied in the control system:

- Unique 1-Wire Interface

- Unique 64-Bit Serial Code Stored in an On-Board ROM

- Power Supply Range is $3.0 \mathrm{~V}$ to $5.5 \mathrm{~V}$

- Measures Temperatures from $-55^{\circ} \mathrm{C}$ to $+125^{\circ} \mathrm{C}$

- $\pm 0.5^{\circ} \mathrm{C}$ Accuracy from $-10^{\circ} \mathrm{C}$ to $+85^{\circ} \mathrm{C}$

- Selected Thermometer Resolution 11 bits which corresponds to $0.125^{\circ} \mathrm{C}$

Fig. 5. Diagram of the temperature controller unit of the blackbody simulator and basic parameters of used temperature sensors 
The controller has ensured to preserve the established temperature with accuracy equal to $0.2^{\circ} \mathrm{C}$ which confirmed measurements performed for different temperatures. In figure 6 , temperature changes during controller operation in $50^{\circ} \mathrm{C}$ and $80^{\circ} \mathrm{C}$ were shown.

a)

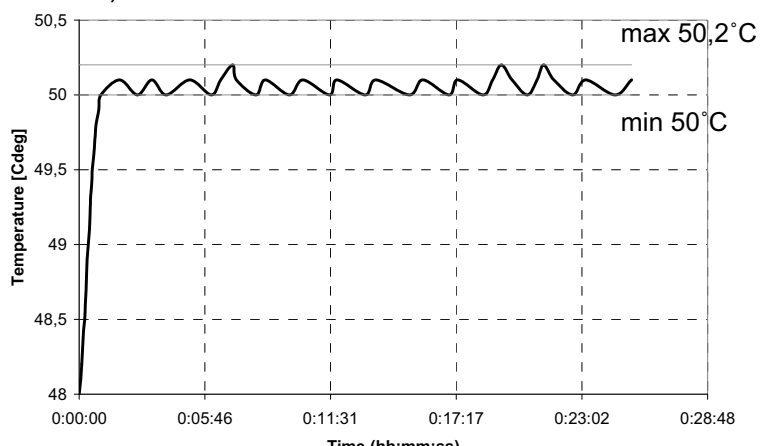

b)

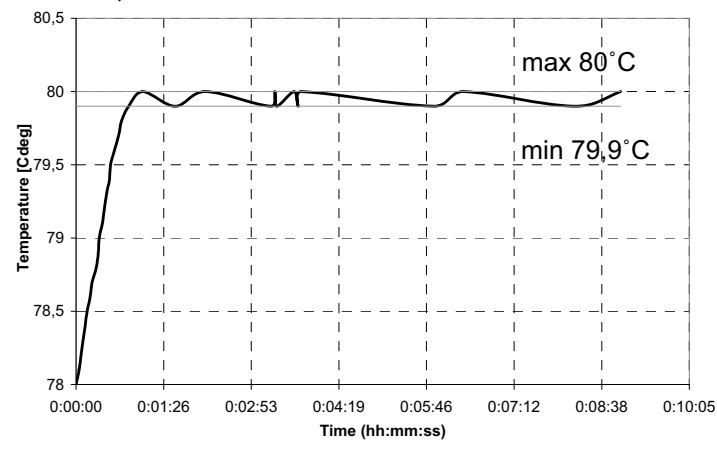

Fig. 6. Temperature measurements of the conic radiator during operation of the temperature controller in a) $50^{\circ} \mathrm{C}$ and b) $80^{\circ} \mathrm{C}$

\section{Thermovision inspection of the black body simulator}

A model of the black body was investigated with the use of a thermovision camera. Series of measurements were done in order to check whether the real emissivity value agreed with computational one and also to estimate the quality and accuracy of the device. Investigations were performed in laboratory of Department of Fundamentals of Machinery Design of Silesian University of Technology were the experimental setup (figure 7) consisting of infrared camera VarioCam by Infratec and a computer with IRBIS software for recording and analysis of thermovision images were prepared.

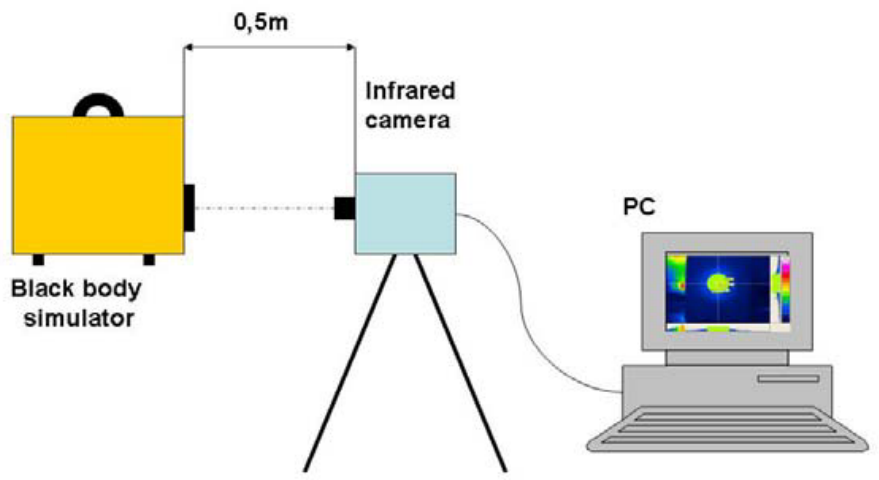

Fig. 7. Experimental setup used for inspection of the blackbody simulator

In figure 8 an exemplary thermovision image of the conical radiator with the temperature profile was presented. It has been clear to observe that the temperature over the aperture surface of the blackbody simulator was distributed uniformly. Some temperature gradients occurred near the edge of the hole.

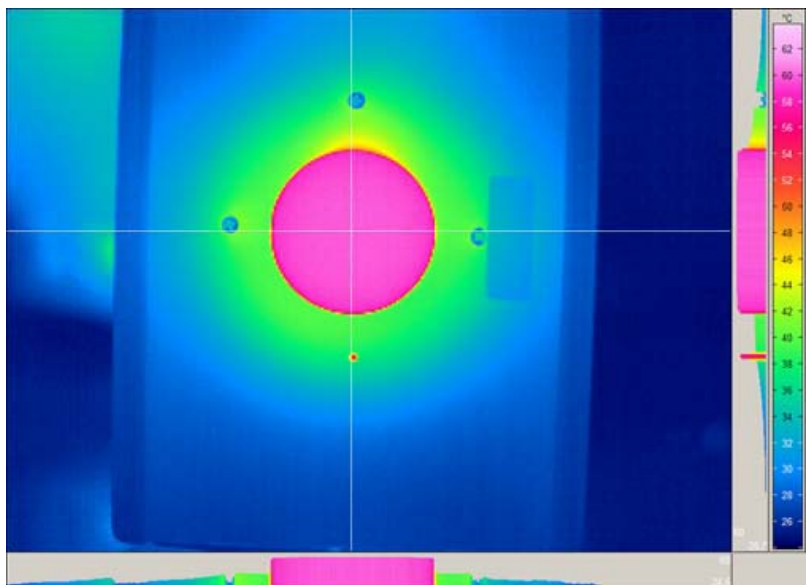

Fig. 8. Infrared image of the black body simulator with horizontal and vertical temperature profiles

In order to evaluate aperture temperature non-uniformity, measurements in some selected points and circular area for different settings of operational temperature were performed (Table 1). 
Table 1. Results of temperature measurements in selected points and the area of aperture of the blackbody

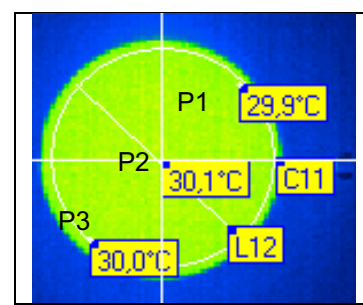

\begin{tabular}{|c|c|c|c|c|c|c|}
\hline \multirow{2}{*}{$\begin{array}{c}\text { Set } \\
\text { temp }\end{array}$} & \multicolumn{2}{|c|}{ Point measurements } & \multicolumn{3}{c|}{ Circle area measurements } \\
\cline { 2 - 7 } & $\mathrm{P} 1$ & $\mathrm{P} 2$ & $\mathrm{P} 3$ & $\min$ & $\max$ & mean \\
\hline 30 & 29,9 & 30,1 & 30 & 29,7 & 30,2 & 30 \\
\hline 40 & 39,5 & 39,6 & 39,5 & 39,2 & 39,9 & 39,6 \\
\hline 50 & 48,9 & 49,2 & 49,1 & 48,7 & 49,5 & 49,2 \\
\hline 60 & 58,4 & 59 & 58,4 & 58,1 & 59,1 & 58,7 \\
\hline 70 & 68 & 68,6 & 68,1 & 67,7 & 68,9 & 68,4 \\
\hline 80 & 77,6 & 78,5 & 77,6 & 77,2 & 78,6 & 78 \\
\hline
\end{tabular}

In figure 9 there was shown a plot of temperature difference between established and measured temperature in selected points. One could observe that the temperature difference was increasing along with the established temperature, thus it could affect a device calibration error. Higher temperature differences have been noticed on the edge of the hole where a temperature gradient occurred. It was shown in figure 8. Stable and smaller temperature differences have been observed in the middle of the aperture where temperature was distributed uniformly.

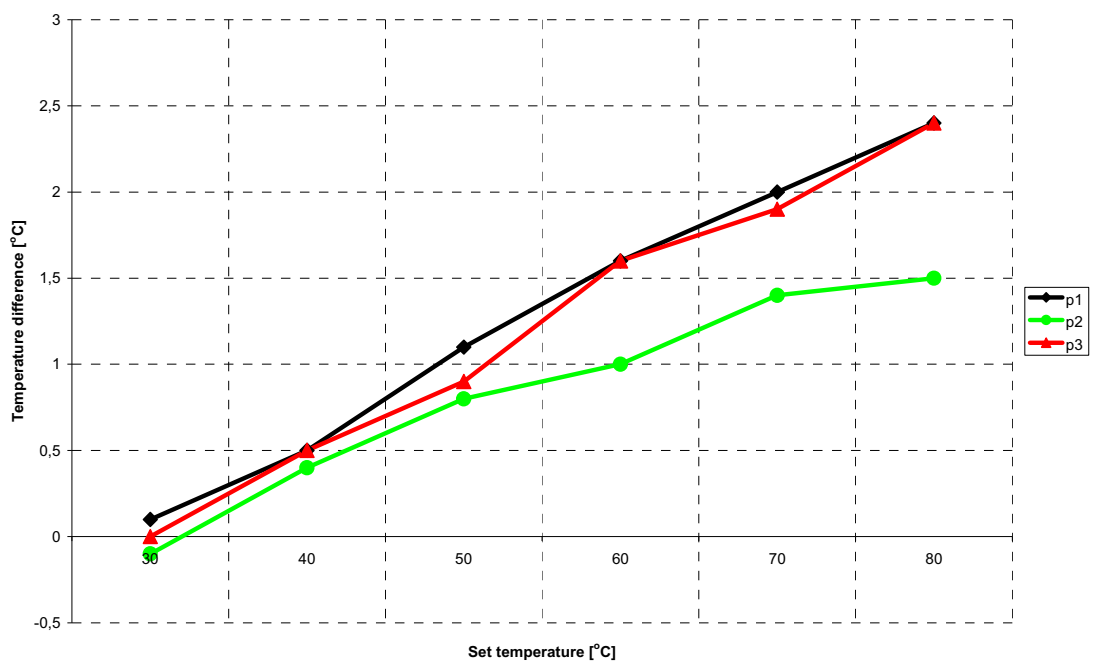

Fig. 9. Plot of temperature differences between established and measured values of temperatures in points p1-p3

In figure 10 the differences between established and measured temperatures into the circular area were graphically presented. Similarly to previous measurement points we have been able to observe that the error between established and measured temperature was growing. Maximum and minimum values indicated that difference between them were constant and changed linearly according to linear growing of the temperature difference.

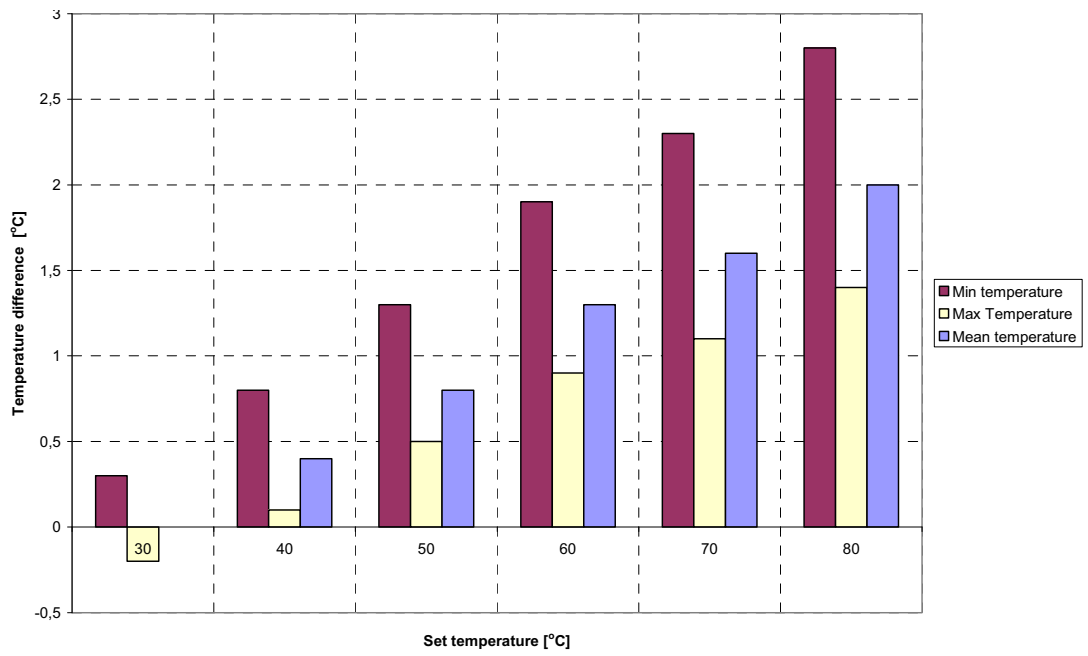

Fig. 10. Plot of temperature differences between established and measured values of temperatures in circular area

In order to compare computational values during the thermovision inspection of the designed and manufactured blackbody simulator aperture emissivity was also estimated. Emissivity was determined for the point located in the center of the hole and for points spread over circular area where all temperature gradients and temperature distribution were going to be flat. During the these tests the reference temperature was established in the device controller, and the value of emissivity was being changed until the temperature in the point or mean temperature 
in the circular area were equal to the established value. One could observe that emissivity values (table 2) were very close to the computational one what confirmed theoretical assumptions as well as correct operation of the blackbody simulator.

Table 2. Results of aperture emissivity measurements in the selected point and circular area

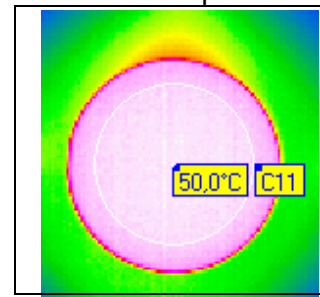

\begin{tabular}{|c|c|c|}
\multirow{2}{*}{ Set temperature } & \multicolumn{2}{|c|}{ Aperture emissivity } \\
\cline { 2 - 3 } & Circular area & Center Point \\
\hline 30 & 0,979 & 0,973 \\
\hline 40 & 0,978 & 0,972 \\
\hline 50 & 0,972 & 0,968 \\
\hline 60 & 0,966 & 0,968 \\
\hline 70 & 0,966 & 0,966 \\
\hline 80 & 0,966 & 0,966 \\
\hline
\end{tabular}

In figure 11, a plot of emissivity changes was presented. It has been clear to observe that emissivity values decreased as set temperature increased. Decreasing of the emissivity could be caused by the previously observed temperature error which increased according to the established temperature.

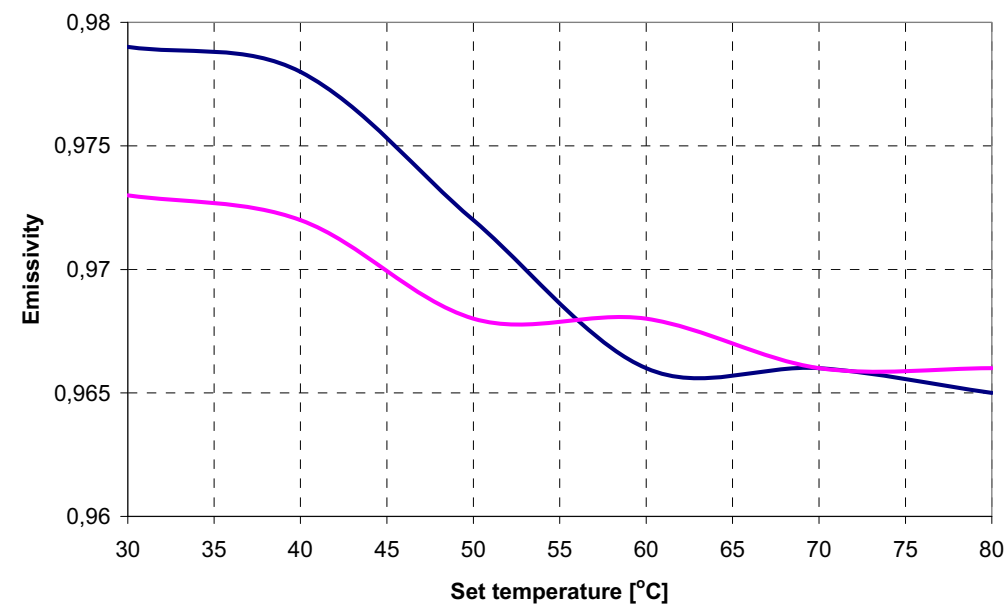

Fig. 11. Plot of emissivity changes as a function of blackbody temperature

\section{Conclusions}

In the article the blackbody simulator designed and manufactured in Department of Fundamentals of Machinery Design of Silesian University of Technology was described. The blackbody simulator has been based on a concept of the conical radiator. Its geometry was assumed on the basis of simple computation and technological aspects. Computational emissivity of the cone aperture was 0.9747 . The device has been equipped with the temperature controller based on a microprocessor. Developed control software allowed to precisely regulate and control of radiator temperature in range from room temperature to $80^{\circ} \mathrm{C}$. The manufactured black body simulator was put to simple tests which aim was to verify whether its emissivity corresponded to the computational one and determine characteristics of the temperature distribution of the aperture.

Due to lack of accurate measurement equipment only preliminary and estimated evaluation of blackbody simulator parameters have been performed. It could be stated that emissivity has been quite good and in whole temperature range it has been higher than 0,96 what corresponded to the computational emissivity value. The temperature distribution on the bottom surface of the cone has been flat. The total cost of all parts, materials and some technological operations which were necessary to produce the simulator was about 50 euros. In comparison to commercial devices we could state that a really cheap device with satisfactory value of the emissivity, good temperature stability and large aperture has been elaborated. However, some modifications of the device construction are necessary to be carried out. Exemplary areas of improvements are to increase emissivity value of the inner surface of the cone radiator, to make temperature range wider, to modify the regulation algorithm and to make the radiator temperature system more accurate. Apart from that precise testing of the device with the use of accurate measurement equipment should be performed what is going to be the aim of further works.

\section{REFERENCES}

[1] G. De Mey, B. Więcek. How to design a black body?. Proceedings of VII Conference Termografia i Termometria w Podczerwieni TTP 2006, Ustroń-Jaszowiec, 16-18 November 2006, Poland.

[2] Web page http://test-therm.com.pl/ir/blackbody.htm

[3] Web page http://www.ci-systems.com/eo/HTMLs/categories.aspx?C2003=12530\&BSP=12529

[4] Web page http://www.temperatures.com/bbf.html 